\title{
Studying the Reflection Spectra of the New Black Hole X-Ray Binary Candidate MAXI J1631-479 Observed by NuSTAR: A Variable Broad Iron Line Profile
}

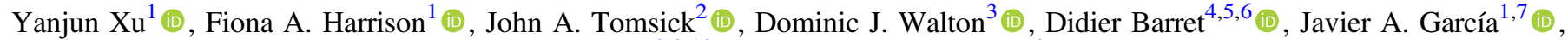 \\ Jeremy Hare ${ }^{2,8,10}$ (10), and Michael L. Parker ${ }^{9}$ (1) \\ ${ }^{1}$ Cahill Center for Astronomy and Astrophysics, California Institute of Technology, Pasadena, CA 91125, USA \\ ${ }^{2}$ Space Sciences Laboratory, 7 Gauss Way, University of California, Berkeley, CA 94720-7450, USA \\ 3 Institute of Astronomy, University of Cambridge, Madingley Road, Cambridge CB3 0HA, UK \\ ${ }^{4}$ Université de Toulouse, 9 Avenue du colonel Roche, BP 44346, F-31028 Toulouse cedex 4, France \\ ${ }^{5}$ CNRS, 9 Avenue du colonel Roche, BP 44346, F-31028 Toulouse cedex 4, France \\ ${ }^{6}$ Institut de Recherche en Astrophysique et Planétologie, 9 Avenue du colonel Roche, BP 44346, F-31028 Toulouse cedex 4, France \\ ${ }^{7}$ Remeis Observatory \& ECAP, Universität Erlangen-Nürnberg, Sternwartstr. 7, D-96049 Bamberg, Germany \\ ${ }^{8}$ NASA Goddard Space Flight Center, Greenbelt, MD 20771, USA \\ ${ }^{9}$ European Space Agency (ESA), European Space Astronomy Centre (ESAC), E-28691 Villanueva de la Cañada, Madrid, Spain \\ Received 2019 August 26; revised 2020 March 5; accepted 2020 March 6; published 2020 April 13
}

\begin{abstract}
We present results from the Nuclear Spectroscopic Telescope Array observations of the new black hole X-ray binary candidate MAXI J1631-479 at two epochs during its 2018-2019 outburst, which caught the source in a disk dominant state and a power-law dominant state. Strong relativistic disk reflection features are clearly detected, displaying significant variations in the shape and strength of the broad iron emission line between the two states. Spectral modeling of the reflection spectra reveals that the inner radius of the optically thick accretion disk evolves from $<1.9 r_{\mathrm{g}}$ to $12 \pm 1 r_{\mathrm{g}}$ (statistical errors at $90 \%$ confidence level) from the disk dominant to the power-law dominant state. Assuming in the former case that the inner disk radius is consistent with being at the innermost stable circular orbit, we estimate a black hole spin of $a^{*}>0.94$. Given that the bolometric luminosity is similar in the two states, our results indicate that the disk truncation observed in MAXI J1631-479 in the power-law dominant state is unlikely to be driven by a global variation in the accretion rate. We propose that it may instead arise from local instabilities in the inner edge of the accretion disk at high accretion rates. In addition, we find an absorption feature in the spectra centered at $7.33 \pm 0.03 \mathrm{keV}$ during the disk dominant state, which is evidence for the rare case that an extremely fast disk wind $\left(v_{\text {out }}=0.067_{-0.004}^{+0.001} c\right)$ is observed in a low-inclination black hole binary, with the viewing angle of $29^{\circ} \pm 1^{\circ}$ as determined by the reflection modeling.
\end{abstract}

Unified Astronomy Thesaurus concepts: Accretion (14); Black hole physics (159); X-ray binary stars (1811); X-ray transient sources (1852)

\section{Introduction}

The majority of Galactic black hole X-ray binaries are found as X-ray transients (Corral-Santana et al. 2016; Tetarenko et al. 2016). These are mostly low-mass X-ray binaries (LMXBs), which are stellar-mass black holes accreting from low-mass donor stars that go into recurrent outbursts due to thermalviscous instabilities in the accretion disk (e.g., Frank et al. 2002). During a typical outburst, lasting from months to years, a black hole X-ray binary displays characteristic evolution in its $\mathrm{X}$-ray spectral and timing properties, which are classified into different spectral states (see Remillard \& McClintock 2006; Belloni \& Motta 2016, for reviews). The outburst usually starts from a low/hard state, transitions to a high/soft state, then returns to the low/hard state at the end of the outburst. In addition, intermediate states are often found close to the time of the state transitions.

The X-ray emission from Galactic black hole X-ray binaries comes primarily from two components: blackbody radiation from the accretion disk and inverse Compton emission from the hot and tenuous corona, and they are believed to be coupled in the framework of the disk-corona model (Haardt \& Maraschi 1991, 1993). Reprocessing of the hard X-ray continuum emission by the optically thick accretion disk also imprints

\footnotetext{
${ }^{10}$ NASA Postdoctoral Program Fellow.
}

characteristic features on the X-ray spectrum, most prominently the ionized $\mathrm{Fe} \mathrm{K} \alpha$ fluorescence emission lines, the $\mathrm{Fe} \mathrm{K}$ absorption edge, and the Compton reflection hump arising from absorption and Compton back-scattering (Guilbert \& Rees 1988; Lightman \& White 1988; Fabian et al. 1989). Relativistic effects at a few gravitational radii from the central black hole distort and blend the reflection features, giving rise to a broad and asymmetric line profile.

Modeling the relativistic disk reflection features helps to probe the physical conditions of the inner accretion flow around the central black hole (see Miller 2007; Fabian \& Ross 2010 for reviews and references within). Notably, by fitting the spectrum with physically self-consistent reflection models, it offers a method to locate the innermost edge of the optically thick accretion disk by measuring the degree of gravitational redshift that modifies the reflection spectrum. Assuming that the innermost disk radius is associated with the innermost stable circular orbit (ISCO) around the black hole, we can directly estimate the spin of the black hole. Recently, with high sensitivity and broadband spectral coverage, the Nuclear Spectroscopic Telescope Array (NuSTAR) detected strong relativistic reflection features in several known or new black hole X-ray binaries or binary candidates, where the broad iron line profiles are resolved (e.g., Tomsick et al. 2014; Miller et al. 2015; Parker et al. 2016; Walton et al. 2017; Xu et al. 2018a, 2018b; Buisson et al. 2019). These high-quality data 
Table 1

NuSTAR Observations of MAXI J1631-479

\begin{tabular}{lccc}
\hline \hline & ObsID & Start Time (UTC) & Exposure (ks) \\
\hline OBS1 & 90501301001 & 2019 Jan 17 02:16 & 7.5 (PART I) \\
& & & 8.8 (PART II) \\
OBS2 & 80401316002 & 2019 Jan 27 16:56 & 10.1 \\
OBS3 & 80401316004 & 2019 Jan 30 01:26 & 14.4 \\
\hline
\end{tabular}

Note. Exposure time is the dead-time-corrected on-source live time for one NuSTAR module, FPMA. Mode 6 data account for about $10 \%$ of the exposure time.

sets of bright black hole X-ray binaries are free from pileup distortions and enable detailed studies of the inner accretion flow of black holes based on the diagnostics of relativistic disk reflection features.

MAXI J1631-479 is a new Galactic black hole X-ray binary candidate discovered when the source went into outburst in late 2018. It is only about 8.9 away from the known X-ray pulsar AX J1631.9-4752. X-ray monitoring observations by MAXI revealed enhanced X-ray emission from an area consistent with the position of AX J1631.9-4752 from 2018 December, but greatly exceeding the maximum historically recorded flux of AX J1631.9-4752 (Kobayashi et al. 2018). We performed two short NuSTAR observations on 2018 December 28 to explore the possibility of another source residing within the MAXI error circle (radius of $0^{\circ}$.17). Indeed, NuSTAR clearly resolved two point sources within the MAXI error region and confirmed that the enhanced X-ray emission came from a bright uncataloged X-ray transient at a flux of about 0.8 Crab (2.0-10.0 keV), which was named MAXI J1631-479 (Miyasaka et al. 2018). The characteristics of the light curve and the shape of the energy spectrum of MAXI J1631-479 are both typical for Galactic X-ray binaries in outburst. In addition, the lack of pulsations and the detection of a strong broad $\mathrm{Fe} \mathrm{K} \alpha$ emission line from the preliminary analysis of the NuSTAR data made the source a strong black hole candidate. Subsequent radio, optical, and X-ray observations have been performed to further investigate the characteristics of this new black hole binary candidate (e.g., Eijnden et al. 2019; Kong 2019; Russell et al. 2019). A radio observation on 2019 January 13 by ATCA suggests the detection of an optically thin radio flare in a soft-state black hole binary (Russell et al. 2019).

\section{Observations and Data Reduction}

The new black hole binary candidate MAXI J1631-479 was observed by NuSTAR (Harrison et al. 2013) on 2019 January 17, 27, and 30 (see Table 1 for details). We reduced the NuSTAR data following standard procedure using NuSTARDAS pipeline v.1.6.0 and CALDB v20170817. The source spectra were extracted from a circular region with the radius of $200^{\prime \prime}$ from the two NuSTAR focal plane modules (FPMA and FPMB). Corresponding background spectra were extracted using polygonal regions from source-free areas in the detectors. We also extracted spectra from mode 6 data following the procedures described in Walton et al. (2016) to maximize the available exposure time. Mode 6 data are taken when an aspect solution is not available from the star tracker on the optics bench (CHU4), but solutions are available from the star trackers on the main spacecraft bus (CHU1,2,3), sufficient for spectral analysis of point sources (see Walton et al. 2016 for further details). MAXI J1631-479 was very bright during the three NuSTAR observations; the dead-time-corrected count rates are $\sim 850-1000 \mathrm{ct} \mathrm{s}^{-1}$ for one module, exceeding the Crab count rate in the NuSTAR band. For spectral analysis, we coadded the FPMA and FPMB spectra from each observation using the addspec tool in HEASOFT v6.19. The NuSTAR spectra were grouped to have a signal-to-noise ratio $(\mathrm{S} / \mathrm{N})$ of 20 per energy bin.

Significant spectral variability was found during the first NuSTAR observation (OBS1; see Figure 1, right panel), when the count rate in the hard X-ray band $(>10 \mathrm{keV})$ increases by a factor of $\sim 3$ and the count rate in the soft X-ray band $(<10$ $\mathrm{keV}$ ) remains roughly constant. For spectral modeling, we separated spectra corresponding to the first and second half of the observation, noted as OBS1 PART I and PART II henceforth. No strong spectral variation was detected during OBS2 and OBS3, which enables a time-averaged spectral analysis. We use the NuSTAR spectrum up to $40 \mathrm{keV}$ for OBS1 PART I, as the spectrum starts to become background dominated above $40 \mathrm{keV}$ due to the weakness of the power-law tail during this period. For OBS2 and OBS3, we ignore spectra in the band of $11-12 \mathrm{keV}$ due to the presence of a narrow dip centered at $11.5 \mathrm{keV}$, which is calibration related and not intrinsic to the source. The apparent dip is weak $(\mathrm{EW} \approx 10 \mathrm{eV})$ and only noticeable in very bright sources with a hard energy spectrum. For other observations, we use NuSTAR spectra in the full energy band of 3-79 keV.

To address the spectral states during our NuSTAR observations in a broader context, we plot the duration of the NuSTAR observations with the long-term MAXI/GSC (Matsuoka et al. 2009) and Swift/BAT (Krimm et al. 2013) monitoring light curves (Figure 1, left panel). The MAXI/ GSC light curve and hardness ratio were produced by the MAXI/GSC on-demand web interface. ${ }^{11}$ We extracted source counts from a circular region with a radius of 1.6 centered on the source position and extracted background counts from a region with a radius of $2^{\circ}$, using auto bright-source exclusion with a minimum exclusion radius of $1^{\circ}$. The Swift/BAT light curve was obtained from the Swift/BAT Hard X-ray Transient Monitor. ${ }^{12}$ We note that AX J1631.9-4752 was found ( $\sim 2 \mathrm{mCrab}$ in $2-10 \mathrm{keV}$ ) by the NuSTAR observation on 2018 December 28 to be faint. Therefore, although MAXI and BAT cannot resolve MAXI J1631-479 and AX J1631.9-4752, the contamination from AX J1631.9-4752 is negligible. From the monitoring light curves, it is clear that NuSTAR OBS1 caught the source in a disk dominant state, when soft X-ray emission from the accretion disk dominates the spectrum. OBS2 and OBS3 were triggered during the phase when the source was undergoing significant spectral hardening, on the declining phase of the outburst.

\section{Spectral Modeling}

We detect strong relativistic disk reflection features in the spectra of MAXI J1631-479. To highlight the relativistic disk reflection features, we fit the NuSTAR spectrum with a disk blackbody model (diskbb; Mitsuda et al. 1984) plus a powerlaw model modified by neutral absorption, TBabs* (diskbb +power law) (Model 1), in XSPEC notation, avoiding energy ranges corresponding to prominent reflection features (4-8 and

\footnotetext{
11 http://maxi.riken.jp/mxondem/

12 https://swift.gsfc.nasa.gov/results/transients/
} 

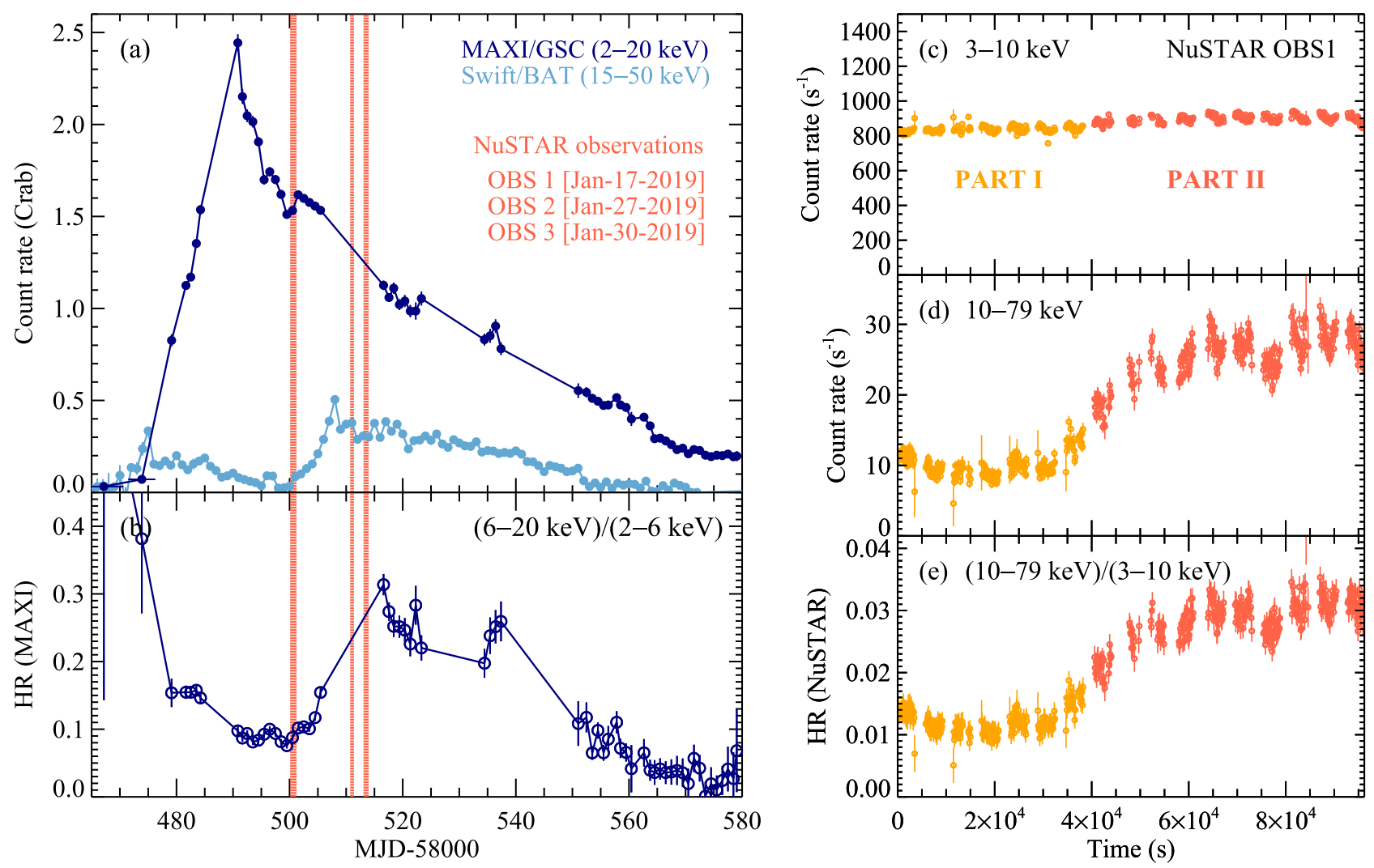

Figure 1. Left panels: (a) long-term MAXI and Swift/BAT monitoring light curves of the 2018-2019 outburst of the new black hole binary candidate MAXI J1631-479. The flux in Crab units is estimated from count rates in the corresponding energy bands. The orange shaded areas mark our NuSTAR observations. (b) Hardness ratio (HR) estimated by count rates in the energy bands of 2-10 and 10-20 keV of MAXI. Right panels: NuSTAR light curves and HR of MAXI J1631-479 for one module (FPMB) during OBS1, when significant flux variation is detected in the hard X-ray band. The NuSTAR count rates have been corrected for dead time.

$12-30 \mathrm{keV})$. In this work, we perform all spectrum modeling in XSPEC v12.9.0n (Arnaud 1996) and use the cross sections from Verner et al. (1996) and abundances from Wilms et al. (2000) in the TBabs neutral absorption model. All uncertainties are reported at the $90 \%$ confidence level. We fit the spectra with an absorbed disk blackbody model, TBabs*diskbb, in XSPEC. As shown in Figure 2, a broad and asymmetric iron line peaking at $\sim 6-7 \mathrm{keV}$ and a Compton hump at $\sim 20-30 \mathrm{keV}$ are evident in the spectral residuals.

Comparing the relative strength of the diskbb and powerlaw components in Figure 2, we note that OBS1 caught MAXI J1631-479 in a state when thermal emission from the accretion disk dominates, consistent with the canonical soft state of black hole binaries. OBS2 and OBS3 were taken when the steep power-law component is dominant, which is similar to the characteristics of the intermediate state/ very high state/steep power-law state in some black hole binaries (e.g., Remillard \& McClintock 2006; Belloni \& Motta 2016; see also the discussion based on INTEGRAL observations in Fiocchi et al. 2020). For simplicity, we henceforth refer to the spectral state during the first and second epochs as the disk dominant and the power-law dominant states, respectively, to avoid possible ambiguities in terms of the state classification.

The broad $\mathrm{Fe} \mathrm{K} \alpha$ line profile clearly varies between the two states: it appears to be broader and stronger in the disk dominant state when compared with the power-law dominant state. As shown in Figure 3(a), the red wing of the line extends to $\sim 4 \mathrm{keV}$ and $\sim 5 \mathrm{keV}$ for the disk dominant and the power- law dominant states, respectively, and the line equivalent width $(\mathrm{EW})^{13}$ decreased from $\sim 180-210$ to $\sim 70 \mathrm{eV}$ from the disk dominant state to the power-law dominant state. We note that by the broad $\mathrm{Fe} \mathrm{K} \alpha$ line here, we are referring to the blurred reflection feature that comes from the iron emission lines and the $\mathrm{Fe} \mathrm{K}$ absorption edge, whose contribution to the reflection feature in the Fe $\mathrm{K}$ band varies with the ionization state (Ross $\&$ Fabian 2005). The shape of the broad $\mathrm{Fe} \mathrm{K} \alpha$ line profile is relatively constant over shorter time intervals (between OBS1 PART I and PART II, and between OBS2 and OBS3), although the spectral continuum varies considerably. Therefore, for more detailed spectral modeling, we fit the spectra of the two states separately and link the relativistic reflection parameters between OBS1 PART I and PART II, and between OBS2 and OBS3.

We plot the evolution of the iron line flux with the power-law flux in the 10-79 keV band during our NuSTAR observations in Figure 3(b), which represent the strength of the observed reflection feature in the Fe $\mathrm{K}$ band and the strength of the hard $\mathrm{X}$-ray illumination from the corona, respectively. The broad iron line flux does not correlate positively with the flux of the nonthermal power-law component, which is in contradiction to the general trend found by previous studies of black hole X-ray binaries observed by RXTE (e.g., Park et al. 2004; Rossi et al. 2005; Reis et al. 2013; Steiner et al. 2016). The ratio of the iron

\footnotetext{
${ }^{13}$ EW and flux of the broad iron line is estimated by adding a Gaussian emission line model to Model 1 with other model parameters fixed at the bestfit values in Model 1.
} 

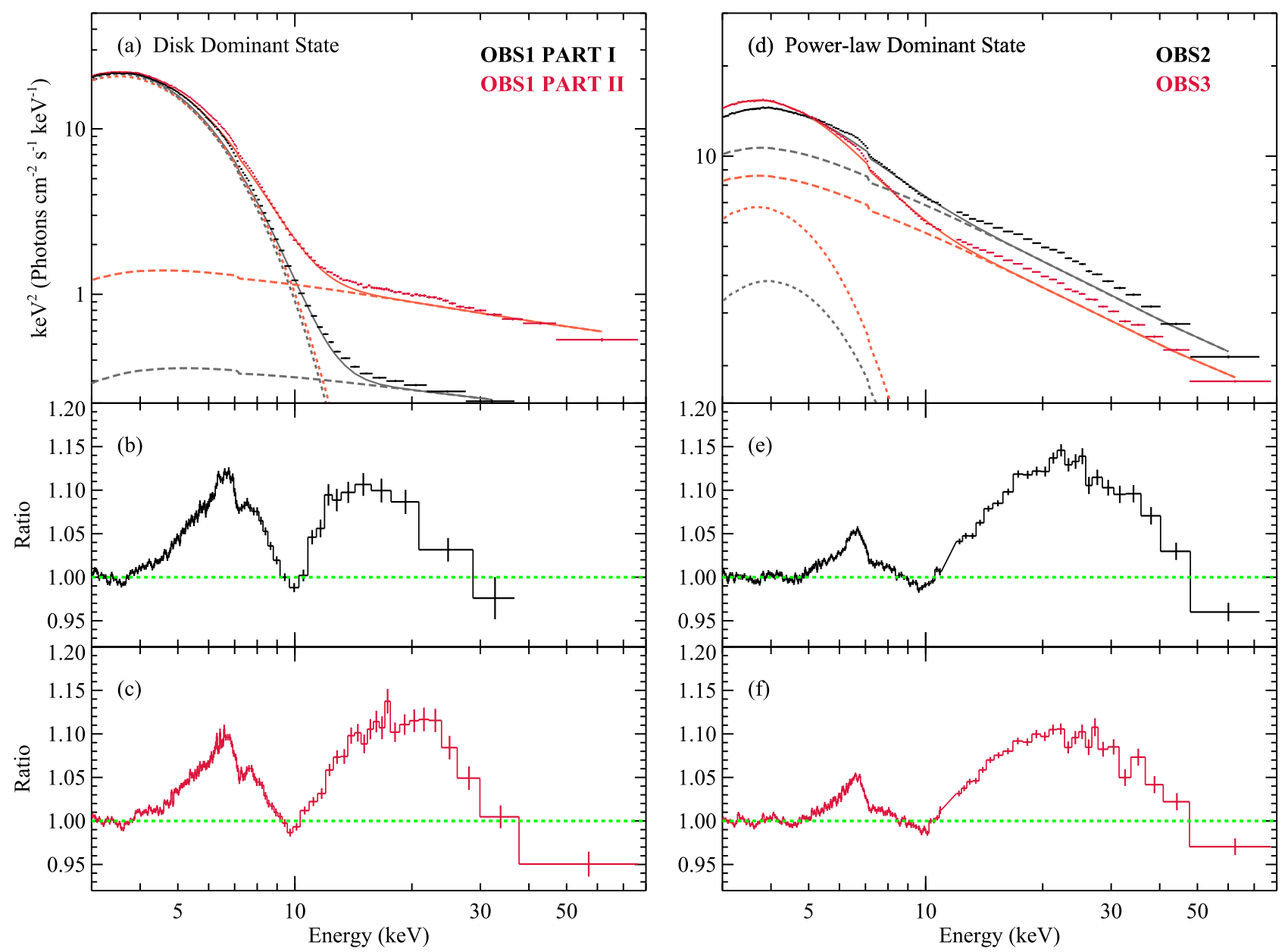

Figure 2. Top panels: unfolded NuSTAR spectra of MAXI J1631-479 in the disk dominant and the power-law dominant state, with the model of a disk blackbody and a nonthermal power-law component modified by neutral absorption, TBabs* (diskbb+powerlaw) (Model 1). The disk blackbody and the power-law model components are plotted in dashed and long-dashed lines, respectively. Middle and bottom panels: strong relativistic reflection features (i.e., a broad iron line and the Compton reflection hump) are shown in the residuals. The spectra are rebinned for display clarity.

line flux to the power-law flux in the $10-79 \mathrm{keV}$ band varies by a factor of $\sim 50$ during our NuSTAR observations. Although the effects of gravitational light bending would possibly produce an anticorrelation, the effect is not predicted to be this large in the lamppost coronal geometry (e.g., Miniutti et al. 2004; Reis et al. 2013). The broad iron line we observed in MAXI J1631-479 during OBS1 PART I is unusually strong when compared with the weakness of the coronal emission, despite reflection features being generally believed to be weak in disk dominant states. This implies that the conventional picture that the apparent reflection features solely originate from reprocessed nonthermal coronal emission may not be true for the case of MAXI J1631-479 in the disk dominant state.

\subsection{Disk Dominant State}

The shape and the strength (EW) of the $\mathrm{Fe} \mathrm{K} \alpha$ line in MAXI J1631-479 remains relatively constant between OBS1 PART I and PART II, despite the large flux variation of the nonthermal emission in the hard X-ray band, implying that the strong and constant thermal disk emission plays a major role in shaping the reflected emission. In this case, the thermal disk component is dominant up to $\sim 10 \mathrm{keV}$ (see Figure 2, left panel), which should provide the majority of high-energy X-ray photons that are able to ionize $\mathrm{Fe} \mathrm{K}$-shell electrons and produce the $\mathrm{Fe} \mathrm{K} \alpha$ emission line (i.e., photons with energies above $7.1 \mathrm{keV}$ ), when transmitting through the disk atmosphere. Thus, thermal disk photons can also cause iron fluorescence and an increase in the observed iron line flux above what would be expected from the coronal illumination alone. Therefore, we propose that this reprocessing of high-energy disk photons could explain the anticorrelation observed in Figure 3(b). This would explain why the iron line flux in the disk dominant state is not correlated with the power-law flux without the need to invoke light-bending effects on the coronal emission. In addition, strong disk emission should have a significant effect on determining the ionization structure of the disk atmosphere.

Therefore, in the disk dominant state of MAXI J1631-479, thermal radiation from the hot accretion disk is important to the reflection process. We use the refbhb (Ross \& Fabian 2007) reflection model to fit the reflection spectra observed here. The refbhb reflection model takes into account the interaction between thermal disk photons and the disk atmosphere by directly including disk emission entering the surface layer from below. Thus, the model is suitable for the disk dominant states of black hole binaries, when the thermal disk emission is hot and strong. The model self-consistently calculates the emergent spectrum that results from the illumination of the hot inner 

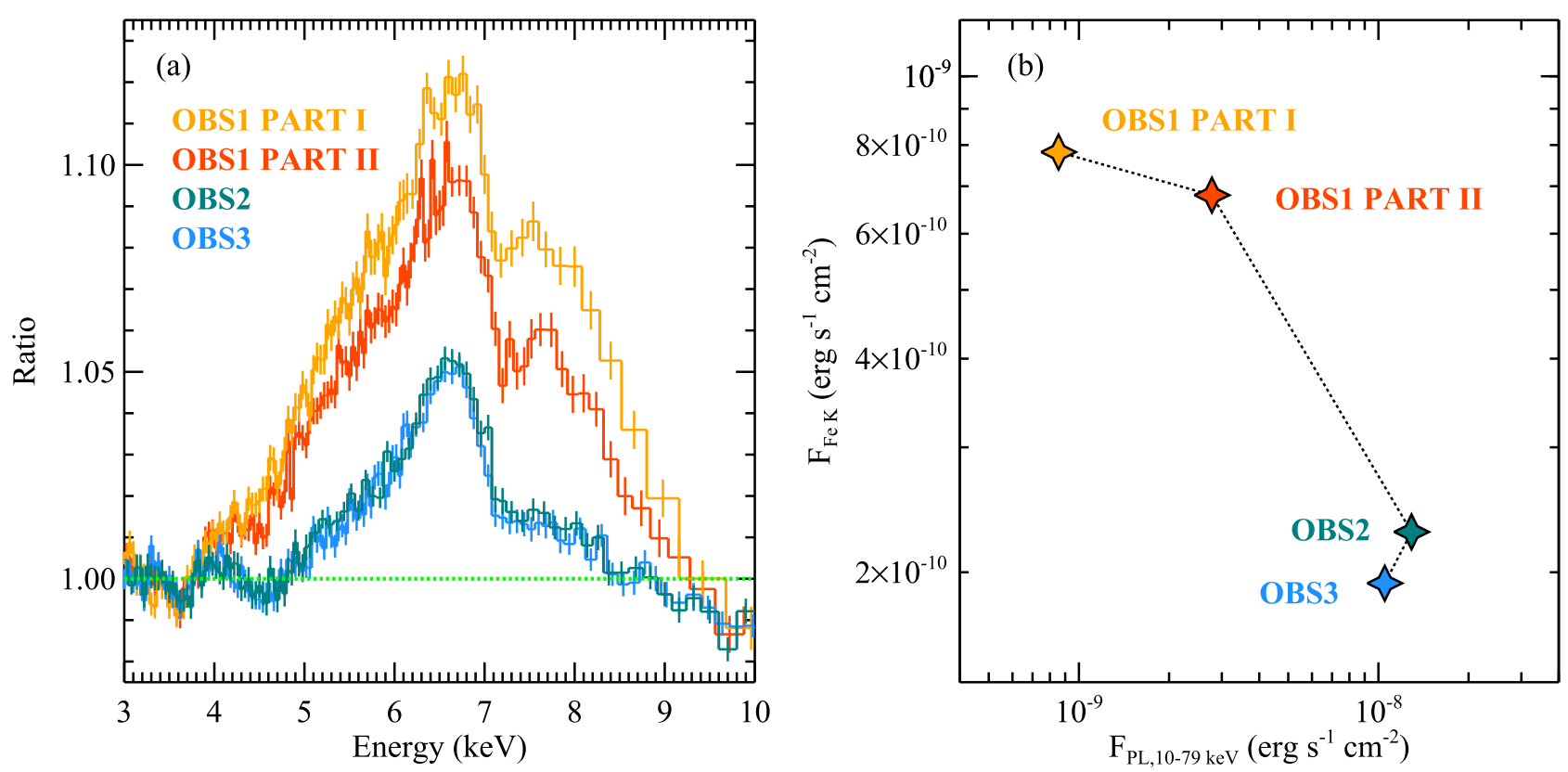

Figure 3. Left panel: zoom-in plot of the Fe K band in the spectral residuals of Figure 2. The spectra are rebinned for display clarity. Right panel: evolution of the Fe K line flux with the hard X-ray flux from the corona; the latter is represented by the flux of the power-law model component in the 10-79 keV band.

portion of a constant-density disk atmosphere and takes into account line broadening due to Compton scattering. The parameters of the model are the number density of hydrogen in the illuminated surface layer, $H_{\mathrm{den}}$, the blackbody temperature of the thermal disk emission entering the disk surface layer from below, $k T_{\mathrm{BB}}$, the power-law index of the coronal emission illuminating the surface layer from above, $\Gamma$, and the flux ratio of the coronal emission illuminating the disk and the thermal emission radiating from the disk, Illum/BB. Elemental abundances are fixed at solar values in the refbhb model.

We convolve the disk reflection component with the relconv_lp model (Dauser et al. 2010, 2013) to measure the relativistic blurring effects on the reflection component. The relconv_lp model assumes an idealized lamppost geometry (i.e., the corona is a point source located on the spin axis of the black hole above the accretion disk). It parameterizes the disk emissivity profile by the height of the corona, $h$, with a lower $h$ corresponding to a steeper disk emissivity profile (e.g., Wilkins \& Fabian 2012; Dauser et al. 2013). We note that even in cases where the lamppost geometry may not be the most realistic assumption, $h$ can still be viewed as a proxy for the general shape of the disk emissivity profile (Dauser et al. 2013), as currently even very high-quality data cannot distinguish between the disk emissivity calculated in the lamppost geometry and that assumed in the form of a broken power law (e.g., Miller et al. 2015; Parker et al. 2015; Xu et al. 2018a). Other parameters of the relconv_lp model are the black hole spin, $a^{*}$, the inner radius, $R_{\mathrm{in}}$, and the inclination, $i$, of the accretion disk.

The total model is set up in XSPEC as TBabs* (relconv_lp*(refbhb+powerlaw)) (Model 2). The powerlaw model component is used here to account for the coronal emission that goes directly toward the observer without being reflected. We link the power-law index, $\Gamma$, in powerlaw and refbhb. As the black hole spin, $a^{*}$, and inner disk radius, $R_{\text {in }}$, are degenerate parameters, we fix $R_{\text {in }}$ at the radius of the ISCO, aiming to obtain a measurement of the black hole spin. All parameters are linked between OBS1 PART I and PART II, except $\Gamma$, Illum/BB, and the normalization of the model components.

As shown in Figure 4(b), Model 2 is able to account for most of the reflection features and greatly improves the fit $\left(\chi^{2} / \nu=1825.8 / 800\right.$, where $\nu$ is the number of degrees of freedom), leaving only a narrow $\mathrm{Fe} \mathrm{K} \alpha$ emission line centered at $6.50 \pm 0.03 \mathrm{keV}$, some excess in the Compton hump region, and a narrow absorption feature centered at $7.33 \pm 0.03 \mathrm{keV}$ in the spectral residuals. The narrow absorption feature can also be clearly seen in Figure 3(a) as a dip in 7-8 keV superposed on the broad iron line profile. We add an unblurred reflection component to fit the weak and narrow iron emission line, which possibly originates from distant reprocessing of the hard X-ray photons. Absorption features in the Fe $\mathrm{K}$ band are commonly associated with blueshifted $\mathrm{FeXXV} / \mathrm{Fe} \mathrm{XXVI} \mathrm{lines,} \mathrm{but} \mathrm{the}$ narrow absorption line complex cannot be resolved by NuSTAR. They are believed to arise from absorption by outflowing material launched from the accretion disk (e.g., Ponti et al. 2012; Díaz Trigo \& Boirin 2016). If the absorption feature at $7.33 \pm 0.03 \mathrm{keV}$ is associated with blueshifted He-like Fe XXV $(6.70 \mathrm{keV})$, it requires an outflowing velocity of $0.094 \pm$ $0.004 c$; or a lower velocity of $0.052 \pm 0.004 c$ if identified with the more ionized H-like Fe XXVI. For a physical modeling of the absorption feature, we include an ionized absorption table model calculated by the XSTAR photoionization code (Kallman \& Bautista 2001). We use the same XSTAR grid as constructed for the soft state of Cygnus X-1 used in Tomsick et al. (2014), the free model parameters are the absorption column density; $N_{\mathrm{H}, \mathrm{XSTAR}}$, the ionization parameter, $\xi$, and the outflowing velocity, $v_{\text {out }}$. We set up the model in XSPEC as TBabs*XSTAR* (relconv_lp* (refbhb ${ }_{1}+$ powerlaw) +refbhb ${ }_{2}$ ) (Model 3).

Model 3 adequately describes the data, leaving no obvious structures in the residuals $\left(\chi^{2} / \nu=1027.9 / 791\right.$; see Figure 4(d)). The addition of an XSTAR grid improves the fit by $\Delta \chi^{2} \sim 160$, indicating that the narrow absorption feature is significantly detected (see the comparison between Figures 4(c) and (d)). 

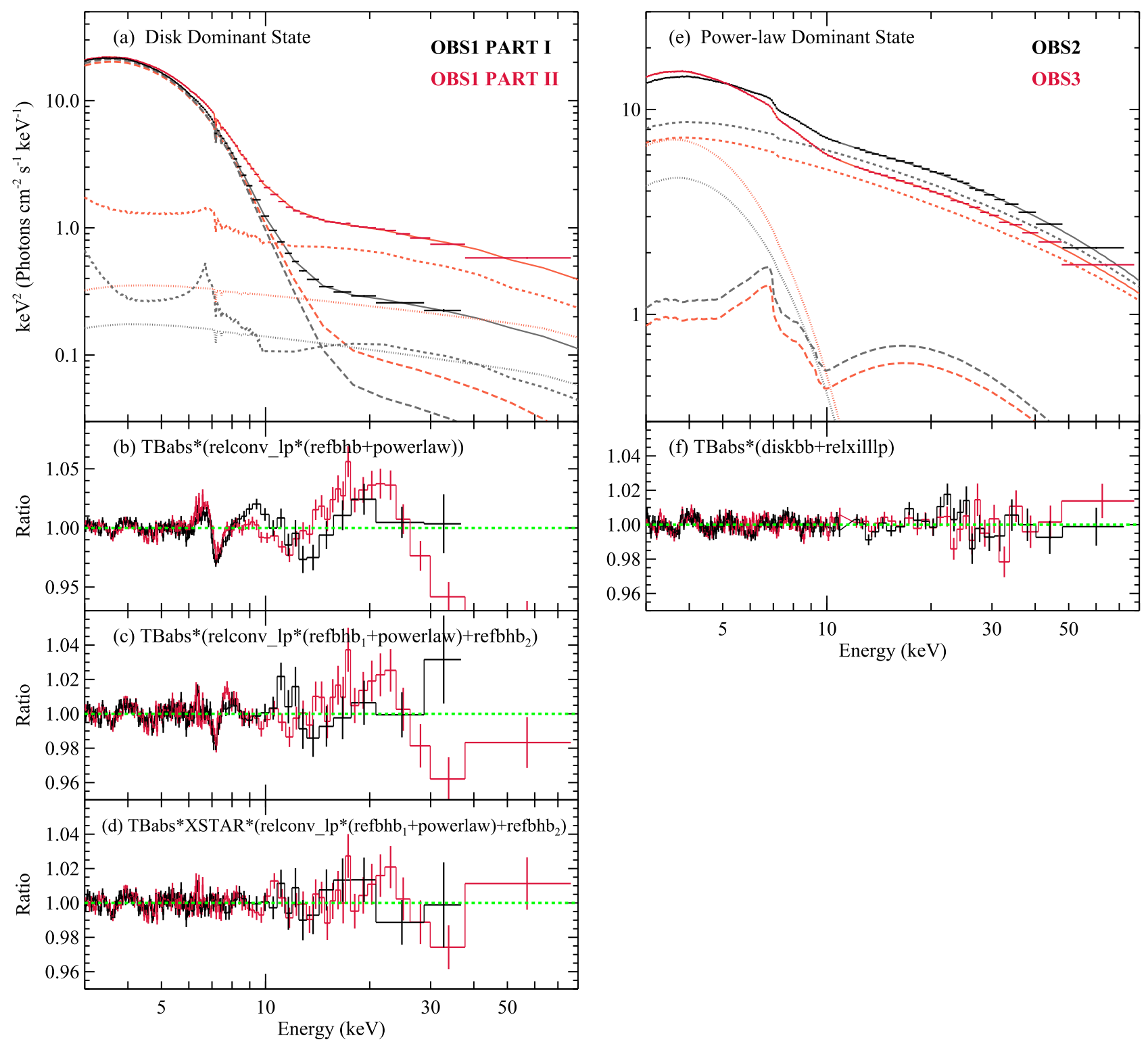

Figure 4. Top panels: unfolded NuSTAR spectra of MAXI J1631-479 with the best-fit models (Model 3 for the disk dominant state, Model 4 for the power-law dominant state). In panel (a), the total model is plotted in solid lines together with the nonthermal coronal emission, powerlaw (dotted lines), the distant reflection

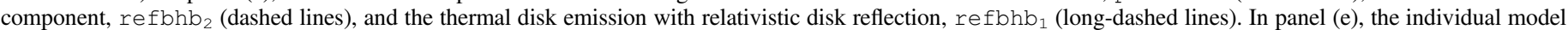
components are the thermal disk emission (dotted lines), the nonthermal coronal emission (dashed lines), and the relativistic reflection component (long-dashed lines). Bottom panels: data/model residuals. The spectra are rebinned for display clarity.

The best fit reveals an outflowing velocity of $v_{\text {out,XSTAR }}=$ $0.067_{-0.004}^{+0.001} c$ for the ionized absorber, which is extremely fast for a disk wind detected in a black hole X-ray binary (e.g., King et al. 2012; Miller et al. 2015). Mostly driven by the clear broad iron line profile, the black hole spin and physical parameters related to the inner accretion flow are well constrained (see Table 2). The fitting results reveal that the central compact object in MAXI J1631-479 is a rapidly spinning black hole with the spin parameter of $a^{*}>0.94$, and the inner accretion disk is viewed at a low inclination of $i=29^{\circ} \pm 3^{\circ}$. By fixing the spin parameter at the maximum value of 0.998 and instead fitting for the inner disk radius, we can get an estimate of the inner disk radius of $R_{\text {in }}<1.9$ $r_{\mathrm{g}}\left(r_{\mathrm{g}} \equiv \mathrm{GM} / c^{2}\right.$, is the gravitational radius). The ionization state of the gas is self-consistently derived by radiative transfer equations in the refbhb model, which is determined by the disk temperature, $k T_{\mathrm{BB}}$, the disk density, $H_{\mathrm{den}}$, and the strength of the illuminating flux from the corona, Illum/BB. We can also get a reasonable constraint on the disk density and temperature based on the spectral modeling. The values are high for the relativistic reflection component, refbhb $b_{1}$, and decrease significantly for the unblurred reflection component, refbhb ${ }_{2}$ (see best-fit parameters in Table 2). This is in accordance with the unblurred reflection occurring at a large distance from the black hole, as both the disk density and temperature are predicted to drop with increasing disk radius in the standard Shakura \& Sunyaev (1973) disk model.

For the spectral fitting above, we linked the disk temperature and the parameters about the relativistic blurring effects between OBS1 PART I and PART II, which is motivated by the constant thermal disk component and the similarity of the observed broad iron line profile. Allowing these parameters to have different values between epochs does not cause any significant change to the fitting results; therefore, we keep them 
Table 2

Spectral Fitting of MAXI J1631-479 (Disk Dominant State)

\begin{tabular}{|c|c|c|}
\hline Parameter & OBS1(PART I) & $\overline{\text { OBS1(PART II) }}$ \\
\hline$N_{\mathrm{H}, \mathrm{TBabs}}\left(\times 10^{22} \mathrm{~cm}^{-2}\right)$ & \multicolumn{2}{|c|}{$3.3_{-0.3}^{+0.4}$} \\
\hline$N_{\mathrm{H}, \mathrm{XSTAR}}\left(\times 10^{22} \mathrm{~cm}^{-2}\right)$ & \multicolumn{2}{|c|}{$1.48_{-0.12}^{+0.15}$} \\
\hline $\log (\xi)_{, \text {XSTAR }}\left(\log \left(\mathrm{erg} \mathrm{cm} \mathrm{s}^{-1}\right)\right)$ & \multicolumn{2}{|c|}{$4.81_{-0.12}^{+0.18}$} \\
\hline$v_{\text {out,XSTAR }}(c)$ & \multicolumn{2}{|c|}{$0.067_{-0.004}^{+0.001}$} \\
\hline$h\left(r_{\mathrm{g}}\right)^{\mathrm{a}}$ & \multicolumn{2}{|c|}{$<3.7$} \\
\hline$a^{*}\left(c J / G M^{2}\right)$ & \multicolumn{2}{|c|}{$>0.94$} \\
\hline$R_{\text {in }}\left(r_{\mathrm{g}}\right)$ & \multicolumn{2}{|c|}{$R_{\mathrm{ISCO}}^{f}\left(<1.9 r_{\mathrm{g}}\right)$} \\
\hline$i\left(^{\circ}\right)$ & \multicolumn{2}{|c|}{$29 \pm 3$} \\
\hline$\Gamma$ & $2.38_{-0.04}^{+0.09}$ & $2.32_{-0.05}^{+0.06}$ \\
\hline Norm(powerlaw) & $9_{-5}^{+12}$ & $17_{-11}^{+25}$ \\
\hline$k T_{\mathrm{BB}, 1}(\mathrm{keV})$ & \\
\hline$H_{\text {den }, 1}\left(\mathrm{~cm}^{-3}\right)$ & \multicolumn{2}{|c|}{$1.7_{-0.4}^{+0.3} \times 10^{21}$} \\
\hline Illum $/ \mathrm{BB}_{1}$ & $0.04_{-0.01}^{+0.04}$ & $0.07_{-0.01}^{+0.05}$ \\
\hline Norm $\left(\text { refbhb }_{1}\right)^{\mathrm{b}}$ & $7_{-1}^{+2}$ & $10 \pm 1$ \\
\hline$k T_{\mathrm{BB}, 2}(\mathrm{keV})$ & \multirow{2}{*}{\multicolumn{2}{|c|}{$\begin{array}{c}0.22_{-0.02}^{+0.06} \\
4_{-1}^{+13} \times 10^{17}\end{array}$}} \\
\hline$H_{\text {den, } 2}\left(\mathrm{~cm}^{-3}\right)$ & & \\
\hline Illum $/ \mathrm{BB}_{2}$ & $0.08_{-0.03}^{+0.06}$ & $0.4_{-0.1}^{+0.3}$ \\
\hline Norm $\left(\right.$ refbhb $\left._{2}\right)$ & $0.7 \pm 0.1$ & $2.6_{-0.3}^{+0.2}$ \\
\hline$\chi^{2} / \nu$ & \multicolumn{2}{|c|}{$1027.9 / 791=1.30$} \\
\hline$F_{3-10 \mathrm{keV}}\left(\mathrm{erg} \mathrm{cm}^{-2} \mathrm{~s}^{-1}\right)^{\mathrm{c}}$ & $2.5 \times 10^{-8}$ & $2.6 \times 10^{-8}$ \\
\hline$F_{10-79 \mathrm{keV}}\left(\mathrm{erg} \mathrm{cm}^{-2} \mathrm{~s}^{-1}\right)^{\mathrm{c}}$ & $9.7 \times 10^{-10}$ & $2.9 \times 10^{-9}$ \\
\hline$F_{0.1-100 \mathrm{keV}}\left(\mathrm{erg} \mathrm{cm}^{-2} \mathrm{~s}^{-1}\right)^{\mathrm{d}}$ & $1.7 \times 10^{-7}$ & $1.6 \times 10^{-7}$ \\
\hline
\end{tabular}

Notes. Parameters marked with a superscript $f$ are fixed during the spectral fitting.

${ }^{\mathrm{a}} r_{\mathrm{g}} \equiv G M / c^{2}$ is the gravitational radius.

$\mathrm{b}$ The refbhb model is normalized based on the power-law incident flux in $1-100 \mathrm{keV}$.

${ }^{c}$ Observed flux in the corresponding energy bands.

${ }^{\mathrm{d}}$ Flux corrected for absorption.

linked to obtain tighter constraints on the key physical parameters of interest. In this case, the strong and hot thermal disk component has a major role in shaping the reflection features rather than the nonthermal emission from the corona, so the relative strength of the different nonthermal components above $20 \mathrm{keV}$ cannot be well constrained (e.g., see the poorly constrained normalization of the powerlaw model in Table 2). Therefore, we do not discuss the physical implications for the fraction of the power-law emission that is reflected by the inner disk, the distant reprocessing material, and the emission that goes directly to the observer as inferred from the best-fit parameters, to avoid overinterpreting the data.

We note that within $3 \sigma$ errors, the flux of the distant reflection component can be either higher or lower than that of the direct power-law component above $20 \mathrm{keV}$, but it is always constrained to be higher than that of the relativistic reflection component. The changes to the quality of the fits and the fit parameters are only negligible if we force the power-law flux to be higher than the flux of refbhb 2 above $20 \mathrm{keV}$. We have tried replacing the powerlaw component in Model 3 with a power-law model with a high-energy cutoff, cutoffpl, a power-law model with a low-energy cutoff, expabs*powerlaw, and a Comptonization model with both low-energy and high-energy rollovers, nthcomp (Zdziarski et al. 1996; Życki et al. 1999), which do not cause any significant difference in

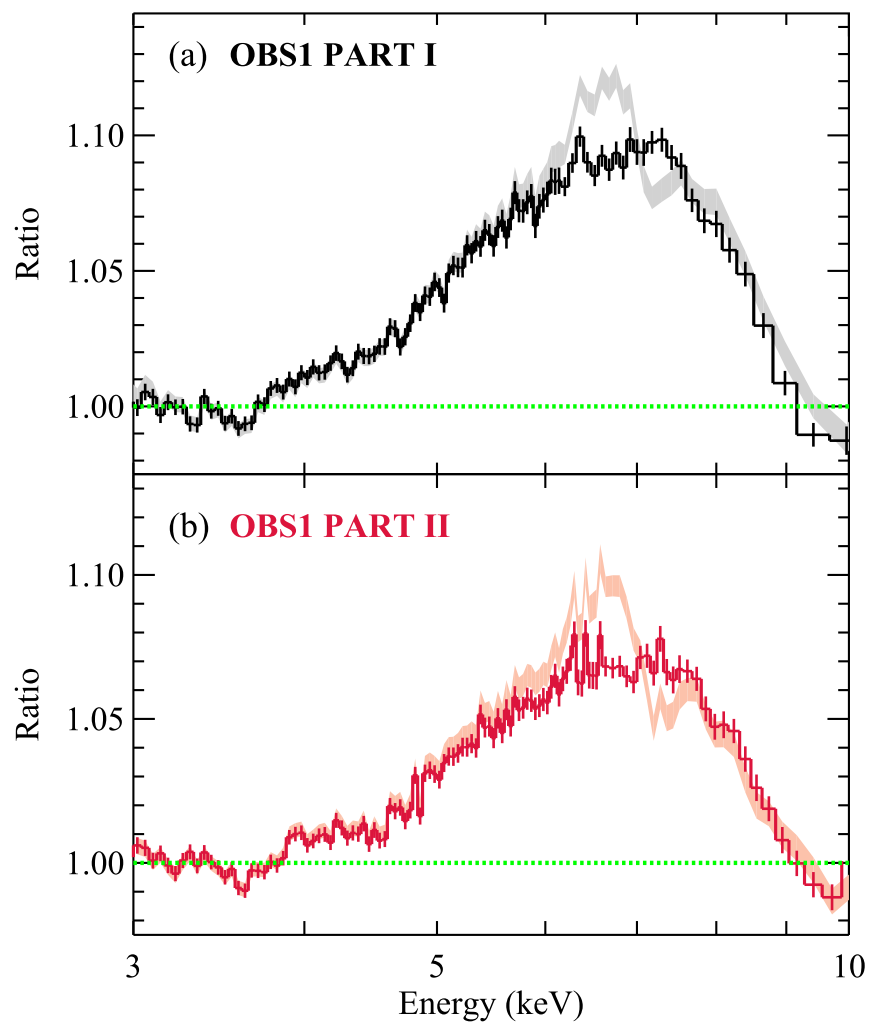

Figure 5. The broad iron line profiles of MAXI J1631-479 observed during the disk dominant state, corrected for the absorption feature at $\sim 7.33 \mathrm{keV}$ and the narrow iron emission line at $\sim 6.50 \mathrm{keV}$. The spectral residuals in the Fe K band here are estimated by adding the XSTAR and refbhb 2 components to Model 1, with their parameters fixed at the best-fit values in Table 2, and fitting the NuSTAR spectra in 3-79 keV, avoiding the energy range of 4-9 keV. For comparison, the line profiles without this correction are plotted in shaded regions.

the fitting results, and the cutoff is not required for the spectral continuum. It is difficult to understand the geometry that could cause the distant reflection to receive more coronal illumination than the relativistic one. We are currently uncertain if this is due to oversimplifications in the current version of the refbhb model, e.g., the fixed iron abundance and the self-consistently calculated ionization assuming a constant accretion rate and disk surface radius (Ross \& Fabian 2007), which might cause a bias in the relative strength of the iron line and Compton reflection hump and make the origin of the refbhb component questionable. If this is the case, the role of the refbh. $\mathrm{b}_{2}$ model component in the spectral fitting might only be phenomenological in some sense. However, as shown in Figure 5, refbhb $\mathrm{b}_{2}$ only contributes to the narrow core of the iron line profile, and thus would not affect the base of the broad iron line profile that we use to deduce the black hole spin.

The single-temperature blackbody description of the accretion disk in the refbhb model is not as realistic as the multicolor blackbody disk model. As discussed in Reis et al. (2008), this simplification might lead to biased values for the inner disk temperature, but would not have a significant effect on the key physical parameters determined by the reflection modeling, such as the inner radius of the accretion disk (or the black hole spin). Modeling the NuSTAR spectra of MAXI J1631-479 above $4 \mathrm{keV}$, mostly only covering the Wien tail of the blackbody distribution, leads to similar fitting results; therefore, we stress that the measurement of a rapid black hole spin here is robust. 
For comparison, we also made an attempt to fit the data with a disk reflection model that does not directly include thermal disk emission, relxillip (see detailed description of the model in Section 3.2). We add a diskbb model to account for the thermal disk component. The fit leads to extreme parameters, a high iron abundance, $A_{\mathrm{Fe}} \sim 5$, and a reflection fraction, $R_{\text {ref }}>10$, for OBS1 PART I, which is driven by the strong iron line but such a high reflection fraction is probably unphysical. The model fails to describe the data, leaving prominent spectral residuals.

\subsection{Power-law Dominant State}

For the power-law dominant state, the flux of the illuminating coronal emission is significantly higher than that of the thermal disk emission, which exceeds the range of parameters covered in the refbhb model. We instead use the relxilllp model (relxill; Dauser et al. 2014; García et al. 2014) to fit the reflection spectra, which is a combination of the ionized disk reflection model xillver (García et al. 2013), which implements the XSTAR code (Kallman \& Bautista 2001) to solve the ionization structure of the disk atmosphere, and the relativistic blurring kernel relconv_lp (Dauser et al. 2010, 2013). The relxilllp model intrinsically includes the illuminating corona emission in the shape of a power law with an exponential high-energy cutoff, $E_{\text {cut }}$. The xillver reflection model is calculated based on the assumption of a slab geometry for the accretion disk and does not include thermal disk photons entering the surface layer of the accretion disk from below. The model assumes a constant density $\left(H_{\mathrm{den}}=10^{15} \mathrm{~cm}^{-3}\right)$ for the surface layer of the accretion disk with the disk temperature fixed at $10 \mathrm{eV}$. Therefore, the model ignores the role of thermal disk emission in determining the physical conditions in the disk atmosphere and thus the emergence reflection spectrum, which suits the case of cool accretion disks in active galactic nuclei (AGNs). The model underestimates the effect of a hot accretion disk on the reflection spectrum for black hole binaries. But it seems to be a reasonable simplification for black hole binaries in the hard and intermediate states, when the thermal disk emission is weak and the nonthermal power-law component dominates the spectrum. The iron abundance, $A_{\mathrm{Fe}}$ (in solar units), and the ionization parameter $\xi$ (defined as $\xi=4 \pi F_{\mathrm{x}} / n$, where $F_{\mathrm{x}}$ is the illuminating flux and $n$ is the gas density) are free parameters in the xillver model.

In addition, we include a separate multicolor disk blackbody component, diskbb, to fit for the thermal emission from the accretion disk. We set up the total model in XSPEC as TBabs* (diskbb+relxilllp) (Model 4). We note that the broad iron line becomes narrower in the power-law dominant state (see Figure 3(a)), implying that the gravitational redshift is weaker in the line emission region, which implies that the Fe $\mathrm{K} \alpha$ emission line is produced at a larger disk radius than that in the disk dominant state. Therefore, we fix the black hole spin at the maximum value of $a^{*}=0.96$ (best-fit value in the disk dominant state), and instead fit for the inner disk radius, $R_{\text {in }}$. We note that the choice of the fixed spin parameter here would not affect the determination of other model parameters. If the accretion disk is truncated at a larger radius than the ISCO, the data would not be sensitive to the black hole spin parameter, as the frame-dragging effect from black holes of different spins would only cause very small differences in the emission line profile produced at large disk radii (e.g., Dauser et al. 2013).
Table 3

Spectral Fitting of MAXI J1631-479 (Power-law Dominant State)

\begin{tabular}{|c|c|c|}
\hline Parameter & OBS2 & OBS3 \\
\hline$N_{\mathrm{H}, \mathrm{TBabs}}\left(\times 10^{22} \mathrm{~cm}^{-2}\right)$ & \multicolumn{2}{|c|}{$3.84_{-0.09}^{+0.10}$} \\
\hline$h\left(r_{\mathrm{g}}\right)$ & \multicolumn{2}{|c|}{$4.3_{-0.3}^{+0.5}$} \\
\hline$a^{*}\left(c J / G M^{2}\right)$ & \multicolumn{2}{|c|}{$0.96^{f}$} \\
\hline$R_{\text {in }}\left(r_{\mathrm{g}}\right)$ & \multicolumn{2}{|c|}{$12 \pm 1$} \\
\hline$i\left(^{\circ}\right)$ & \multicolumn{2}{|c|}{$29 \pm 1$} \\
\hline$\Gamma$ & $2.45 \pm 0.02$ & $2.51 \pm 0.02$ \\
\hline$E_{\text {cut }}(\mathrm{keV})$ & $110 \pm 11$ & $156 \pm 22$ \\
\hline $\log (\xi)_{, \text {ref }}\left(\log \left(\mathrm{erg} \mathrm{cm} \mathrm{s}^{-1}\right)\right)$ & \multicolumn{2}{|c|}{$3.56_{-0.09}^{+0.15}$} \\
\hline$A_{\mathrm{Fe}}($ solar $)$ & \multicolumn{2}{|c|}{$1.0_{-0.1}^{+0.3}$} \\
\hline$R_{\text {ref }}$ & \multicolumn{2}{|c|}{0.49} \\
\hline Norm (relxilllp) & $0.8 \pm 0.1$ & $0.8 \pm 0.1$ \\
\hline$k T_{\text {in }}(\mathrm{keV})$ & $1.19_{-0.02}^{+0.01}$ & $1.14 \pm 0.01$ \\
\hline Norm (diskbb) & $454_{-20}^{+32}$ & $859_{-27}^{+38}$ \\
\hline$\chi^{2} / \nu$ & \multicolumn{2}{|c|}{$1669.6 / 1491=1.12$} \\
\hline$F_{3-10 \mathrm{keV}}\left(\mathrm{erg} \mathrm{cm}^{-2} \mathrm{~s}^{-1}\right)$ & $2.3 \times 10^{-8}$ & $2.3 \times 10^{-8}$ \\
\hline$F_{10-79 \mathrm{keV}}\left(\mathrm{erg} \mathrm{cm}^{-2} \mathrm{~s}^{-1}\right)$ & $1.3 \times 10^{-8}$ & $1.1 \times 10^{-8}$ \\
\hline$F_{0.1-100 \mathrm{keV}}\left(\mathrm{erg} \mathrm{cm}^{-2} \mathrm{~s}^{-1}\right)$ & $2.5 \times 10^{-7}$ & $2.5 \times 10^{-7}$ \\
\hline
\end{tabular}

Model 4 fits the NuSTAR spectra well and leaves no prominent structures in the spectral residuals $\left(\chi^{2} / \nu=1669.6 / 1491=\right.$ 1.12; see Figure 4(f)). The fitting results confirm that the source is not in a canonical hard state during the time of the NuSTAR observation, despite the significant spectral hardening observed in the monitoring light curves in Figure 1(b). From the best-fit parameters (see Table 3), we find the disk temperature is still high and the spectral slope remains soft in the power-law dominant state. The spectral hardening is caused by a large change in the relative strength of the thermal disk and the power-law component; the disk fraction of the total flux in $0.1-100 \mathrm{keV}$ decreases from $\sim 60 \%$ to $\sim 10 \%$ from the disk dominant to the power-law dominant state.

In terms of the reflection parameters, the best-fit results of Model 4 (see Table 3) indicate significant truncation of the inner edge of the accretion disk at $R_{\text {in }}=12 \pm 1 r_{g}$, larger than the ISCO radius for any given black hole spin (the ISCO radius depends on the black hole spin, $R_{\mathrm{ISCO}}=1.235 r_{\mathrm{g}}$ for $a^{*}=0.998, R_{\mathrm{ISCO}}=6 r_{\mathrm{g}}$ for $a^{*}=0$, and $R_{\mathrm{ISCO}}=\sim 9 r_{\mathrm{g}}$ for $a^{*}=-0.998$; Bardeen et al. 1972; Thorne 1974). The disk inclination determined by the reflection method is driven by the relativistic Doppler shift of the iron line, which is sensitive to the blue wing of the line profile (e.g., Brenneman \& Reynolds 2006). The inclination of the inner accretion disk is measured to be $29^{\circ} \pm 1^{\circ}$, which agrees well with the value obtained in the disk dominant state from the refbhb model. The iron abundance measured by the relxilllp model is consistent with the solar value, $A_{\mathrm{Fe}}=1.0_{-0.1}^{+0.3}$, which is also in agreement with the value of elemental abundances fixed in the refbhb model. We note that certain systematic uncertainties could be introduced when fitting the spectra with different reflection models (e.g., see the discussion in Middleton et al. 2016), but the good agreement we find in $i$ and $A_{\mathrm{Fe}}$ determined by the refbhb and relxillip reflection models indicates such uncertainties are probably minimal here.

In addition, we find a reflection fraction of $R_{\mathrm{ref}}=0.49$ for the power-law dominant state, significantly lower when compared 

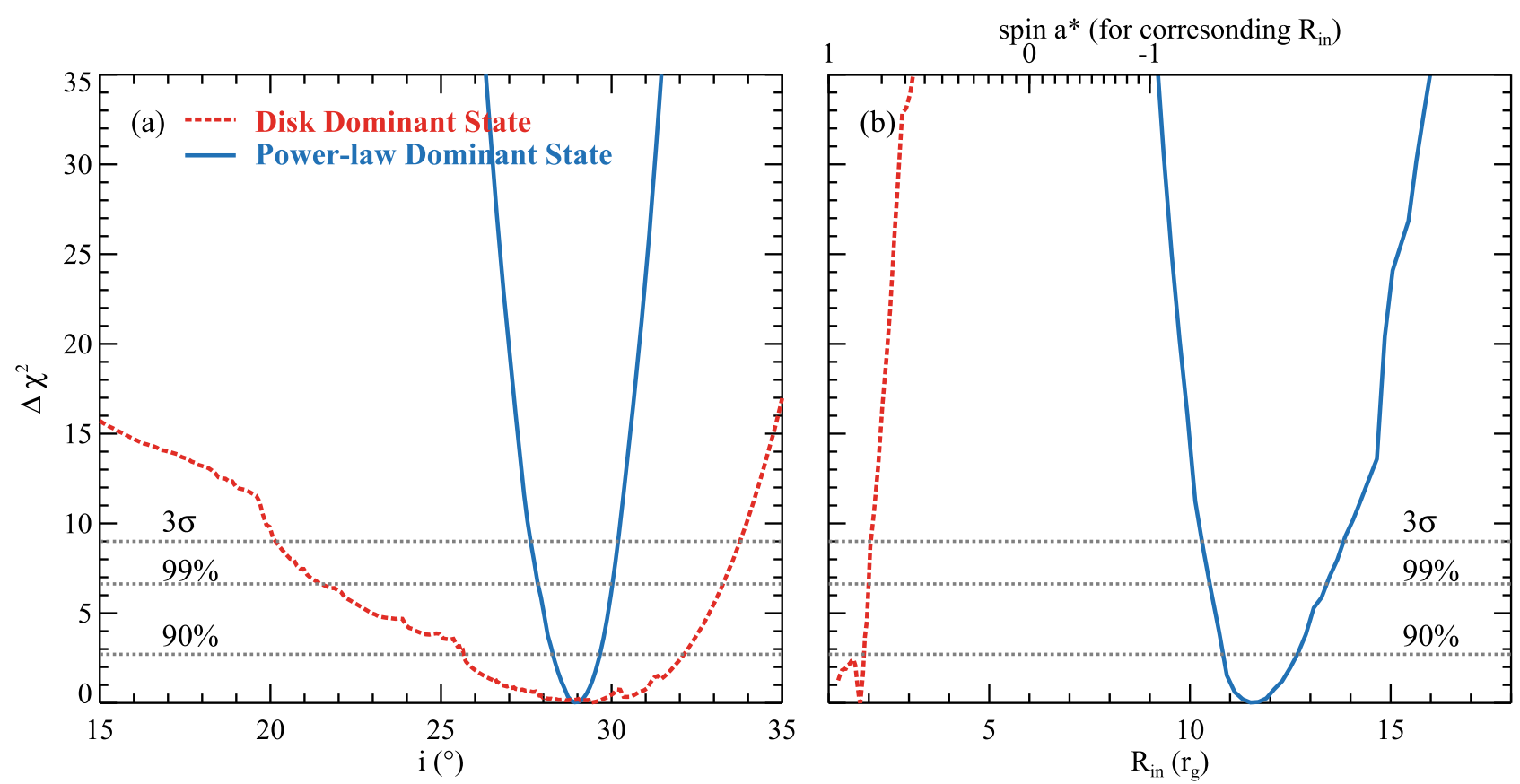

Figure 6. $\Delta \chi^{2}$ plots for the black hole spin parameter $a^{*}$ and inner accretion disk radius $R_{\mathrm{in}}$. The constraints for the disk dominant state and power-law dominant state are marked by the red dashed and blue solid lines. The horizontal lines indicate the $90 \%, 99 \%$, and $3 \sigma$ confidence levels for one parameter of interest.

with that of other black hole binaries displaying strong reflection features (e.g., Miller et al. 2018; Xu et al. 2018a, 2018b). In the relxilllp model, the value is defined as the ratio of the coronal intensity illuminating the disk to that reaching the observer, selfconsistently calculated by the model in the lamppost geometry (Dauser et al. 2016). Leaving the reflection fraction as a free parameter does not cause any significant change to the fit, indicating a truncated accretion disk is indeed required by the shape of broad iron profile. The low reflection fraction is consistent with the relative weakness of the broad iron line in the power-law dominant state, which is a natural outcome of the scenario where the accretion disk is truncated, as X-ray photons would be lost in the gap between the black hole and the inner edge of the truncated accretion disk, without being reprocessed by the optically thick accretion disk.

\section{Discussion}

We have performed detailed modeling of the reflection spectra of the new black hole binary candidate MAXI J1631-479 during its 2018-2019 outburst, which displays a clear variation in the $\mathrm{Fe} \mathrm{K} \alpha$ emission line profile between the two epochs observed by NuSTAR. The inclination of the inner part of the accretion disk is measured to be low in MAXI J1631-479 ( $i \approx 29^{\circ}$, closer to being face-on than edge-on). Therefore, we probably have a relatively clear view of the inner regions around the black hole without being blocked by, e.g., a geometrically thick accretion disk. Detailed modeling of the reflection spectra suggests that the variation of the broad iron line profile is caused by a change in the radius of the inner edge of the optically thick accretion disk, $R_{\mathrm{in}}$, from $<1.9 r_{\mathrm{g}}$ to $12 \pm 1 r_{\mathrm{g}}$, which consistently explains the changes in the iron line width and strength (see constraints of the inclination and the inner disk radius in Figure 6).

Assuming that the small inner disk radius measured during the first epoch is associated with the ISCO radius of the black hole, we can get an estimate of the spin of the black hole of $a^{*}>0.94$, close to the maximum value. The high spin measured indicates that the central compact object in MAXI J1631-479 is likely a black hole instead of a neutron star. In accreting neutron stars, the inner edge of the accretion disk is observed to be truncated at a typically larger radius of $\sim 6-15 r_{\mathrm{g}}$, due to either the magnetic field of the neutron star or the existence of a solid stellar surface (e.g., Cackett et al. 2010; Ludlam et al. 2017, 2019). During the second epoch, however, the inner radius measured via the reflection method becomes significantly larger, which suggests a change in the accretion mode in MAXI J1631-479: the innermost part of the optically thick accretion disk is replaced by an optically thin accretion flow, which is unable to produce clear reflection features inside of the disk truncation radius.

\subsection{The Iron Line Profile and Disk Truncation}

The inner edge of the accretion disk around black holes is predicted to vary with the mass accretion rate, which is inferred from the source luminosity, in units of the Eddington luminosity. The mass accretion rate is thought to influence the disk surface density and thus determine the transitional inner edge of the accretion disk where the matter becomes thin to Thomson scattering, defined as the "reflection edge" (e.g., Krolik \& Hawley 2002). When the accretion rate is high, the optically thick accretion disk is predicted to reach down to the ISCO, and its innermost part would be replaced by an optically thin advection-dominated accretion flow at low accretion rates (e.g., Esin et al. 1997, 1998). Different theoretical models have been proposed to discuss the mechanisms facilitating the disk truncation (e.g., Narayan \& Yi 1995; Honma 1996; Meyer et al. 2000; Yuan \& Narayan 2004). However, what triggers disk truncation in accreting black hole systems and the typical disk truncation radius is still debated.

From observations, the outburst of a black hole binary is considered ideal for exploring the relationship between the accretion disk inner disk radius and the accretion rate, as its 
X-ray luminosity varies by several orders of magnitude during a typical outburst. Recently, there have been numerous observational campaigns of black hole X-ray binaries aiming to use the reflection method to track the evolution of the inner accretion disk radius (e.g., Fürst et al. 2015; Walton et al. 2016, 2017; Xu et al. 2017; Buisson et al. 2019; García et al. 2019; Kara et al. 2019). However, in most cases, the change in the inner disk radius measured at multiple epochs is only marginally significant and can often be considered to be consistent within errors. So far, the best cases regarding variations in the $\mathrm{Fe} \mathrm{K} \alpha$ line profile are the narrow lines detected in the very low states of GX 339-4 (Tomsick et al. 2009) and V404 Cygni (Motta et al. 2017), given that relativistically broadened lines are known to be present in their high states (e.g., Parker et al. 2016; Walton et al. 2017). We note that in the previous cases the $\mathrm{Fe} \mathrm{K} \alpha$ lines are sufficiently narrow and symmetric that, they do not require general relativity effects to explain, signaling a line emission region at a large distance of $\gtrsim 10^{2}-10^{3} r_{\mathrm{g}}$. As proposed in Motta et al. (2017), it is possible that they arise from reprocessing by distant obscuring material, making them an analog to obscured AGNs. Thus, it is debated whether they constrain the inner edge of the accretion disk. The possibility of the existence of two reflection zones (inner accretion disk and distant reprocessing material) is also supported by the narrow core found on top of the broad $\mathrm{Fe} \mathrm{K} \alpha$ emission line profile in several black hole binaries (e.g., Walton et al. 2017; Miller et al. 2018; Tomsick et al. 2018; Xu et al. 2018a, 2018b). In general, high$\mathrm{S} / \mathrm{N}$ detections of a relativistically broadened $\mathrm{Fe} \mathrm{K} \alpha$ emission line changing in line width, which could be used as a strong evidence for a variable inner accretion disk radius in a black hole X-ray binary, are still lacking.

In this work, we obtained high-S/N NuSTAR spectra of the new black hole binary candidate MAXI J1631-479, revealing a broad $\mathrm{Fe} \mathrm{K} \alpha$ emission line variable in line width and strength. Spectral modeling suggests a change in the inner disk radius from $R_{\mathrm{in}}<1.9 r_{\mathrm{g}}$ to $R_{\mathrm{in}}=12 \pm 1 r_{\mathrm{g}}$ between the two epochs observed by NuSTAR, despite the small variance in the bolometric luminosity ${ }^{14}$ of the source at $L_{\text {bol }} \sim$ $\left(1.3 \times 10^{39}-1.9 \times 10^{39}\right) \times(D / 8 \mathrm{kpc})^{2} \mathrm{erg} \mathrm{s}^{-1}$. The Eddington rate at the time of the observations is uncertain, as both the black hole mass and distance are currently unknown. Assuming a typical source distance of $8 \mathrm{kpc}$ and black hole mass of $10 M_{\odot}$, we estimate the Eddington rate of $L / L_{\text {Edd }} \sim$ $(100 \%-150 \%) \times\left(10 M_{\odot} / M\right) \times(D / 8 \mathrm{kpc})^{2}$. Our spectral analysis of MAXI J1631-479 indicates that it is possible for the accretion disk to become significantly truncated at this high accretion rate. In addition, the results suggest that in the case of MAXI J1631-479, the change in the accretion mode between the two epochs is not driven by a variation in the global accretion rate of the system.

At high accretion rates, it is predicted that disk truncation could be triggered by thermal instabilities, due to the dominance of radiation pressure in the inner accretion disk (e.g., Takeuchi \& Mineshige 1998; Gu \& Lu 2000; Lu et al. 2004). Observational evidence for the temporal disappearance of the innermost part of the accretion disk, believed to be associated with the Lightman \& Eardley (1974) instability, has been found in the black hole X-ray binary GRS $1915+105$ (e.g., Belloni et al. 1997) and V404 Cygni (Walton et al. 2017),

\footnotetext{
14 The bolometric luminosity is estimated from the absorption-corrected flux in 0.1-100 keV from the best-fit spectral models.
}

which are known to be accreting close to the Eddington limit. In the case of GRS $1915+105$, the disappearance and followup replenishment of the inner accretion disk have been reported to be associated with the formation of relativistic expanding clouds and jet ejections (e.g., Pooley \& Fender 1997; Mirabel et al. 1998).

\subsection{The Spectral Continuum}

Based on the shape of the spectral continuum, the spectral state during the second epoch is similar to the very high/steep powerlaw state reported in some black hole X-ray binaries, when the luminosity is high and the spectrum is dominated by a steep power-law component (e.g., Remillard \& McClintock 2006; Belloni \& Motta 2016). There is evidence that the inner accretion disk in black hole X-ray binaries is sometimes truncated at the very high state. During the very high state, the constant disk emission area inferred from the relation between the observed temperature and disk luminosity breaks down (e.g., Kubota \& Done 2004; McClintock \& Remillard 2006). Detailed modeling of the thermal disk component, taking into account the coupled energetics of the disk and corona, also reveals that the inner disk radius in the very high state is larger than the location of the ISCO (e.g., Kubota \& Done 2004; Done \& Kubota 2006; Tamura et al. 2012). By modeling the disk reflection spectra, we have measured a significantly truncated disk in MAXI J1631-479 during the second epoch, supporting this interpretation of the disk structure in the very high state based on previous studies of the thermal disk component. However, because the distance and black hole mass of MAXI J1631-479 are currently unknown, we are unable to obtain an accurate measurement of the inner disk radius by modeling the thermal disk component and directly comparing that with the value obtained from the reflection method.

For an approximate estimation, we tried fitting the spectra with the model, TBabs*simpl*diskbb, only aiming to describe the shape of the spectral continuum without selfconsistently modeling the reflection features. We use the simpl convolution model (Steiner et al. 2009b) to account for the effect that a fraction of the input disk seed photons are redistributed by Comptonization into a power-law component (e.g., Nakahira et al. 2018; Shidatsu et al. 2019). We only model the disk dominant state spectra, as the simplified Comptonization model, simpl, is not applicable to the power-law dominant state based on the model assumptions (Steiner et al. 2009a). In order to reveal the intrinsic temperature and luminosity of the thermal disk component during the power-law dominant state, more complicated calculations may be required depending on the disk-corona geometry (e.g., Kubota \& Done 2004; Done \& Kubota 2006; Gierliński et al. 2008). The spectral fit yields $k T_{\text {in }} \sim 1.0 \mathrm{keV}, N_{\text {diskbb }} \sim 4000$ for the disk dominant state. Based on the definition of the normalization parameter of

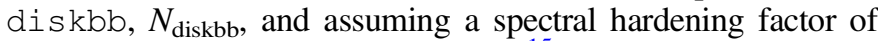
$f=1.7$ (Shimura \& Takahara 1995), ${ }^{15}$ we estimate the inner disk radius to be $\sim 10 \times\left(10 M_{\odot} / M\right) \times(D / 8 \mathrm{kpc}) r_{\mathrm{g}}$. We note that there are several possible uncertain factors regarding the thermal disk modeling here. It has been proposed that the spectral hardening factor is likely to be variable (e.g., Merloni et al. 2000; Salvesen et al. 2013; Davis \& El-Abd 2019). Also,

\footnotetext{
$15 R_{\mathrm{in}, \mathrm{km}}=f^{2} D_{10 \mathrm{kpc}} \sqrt{N_{\mathrm{diskbb}} / \cos i}$, where $R_{\mathrm{in}}$ is the inner disk radius in kilometers, $f$ is the spectral hardening factor, $D_{10 \mathrm{kpc}}$ is the distance to the source in units of $10 \mathrm{kpc}, N_{\text {diskbb }}$ is the normalization of the dis kbb model, and $i$ is the inner disk inclination. We assume a disk inclination angle of $i=30^{\circ}$ for MAXI J1631-479 when estimating $R_{\text {in }}$
} 
at high accretion rates, the thermal disk emission deviates from that of an idealized thin accretion disk, which makes the inner disk radius inferred by a thin-disk model problematic at high luminosities (e.g., McClintock et al. 2006; Straub et al. 2011).

It is also interesting to consider how the reflection component corresponds to changes of the spectral continuum. Statistical studies of the reflection component in AGNs reveal that disk reflection tends to be less variable than the illuminating continuum (e.g., Miniutti et al. 2003; Miller et al. 2007; Parker et al. 2014). From our observations of MAXI J1631-479, we find that the reflection component stays constant across short time intervals (between OBS1 PART I and II, and between OBS2 and OBS3); the variation of the corona emission has only a negligible effect on the shape of the broad iron line. Significant variation in the reflection spectra occurs at the time of state transition, which arises from dynamical changes in the disk structure. However, considering the fact that in several black hole $\mathrm{X}$-ray binaries previous studies show that the broad iron line remains relatively unchanged in different spectral states (e.g., Reis et al. 2008; Walton et al. 2012; Parker et al. 2015), it is possible that the significant change in the shape of the broad iron line we find in MAXI J1631-479 is a peculiar case.

\subsection{Fast Disk Wind with a Narrow Opening Angle}

Disk winds are believed to be ubiquitous in X-ray binaries and important to the accretion process, which might trigger instabilities in the accretion flow (Begelman et al. 1983) and even lead to accretion state changes (Shields et al. 1986). Disk wind launched in X-ray binaries is generally known to have an equatorial geometry, flowing radially at small angles above the accretion disk. Among LMXBs, blueshifted ionized absorption lines in the X-ray spectrum signaling outflowing material are preferentially found in systems viewed close to edge-on (e.g., Ponti et al. 2012; Díaz Trigo \& Boirin 2016), the majority being "dippers" with an inclination estimate of $60^{\circ}-80^{\circ}$ (Frank et al. 1987).

From the NuSTAR observations of MAXI J1631-479, we find a blueshifted ionized absorption feature in the Fe $\mathrm{K}$ band in the disk dominant state, which disappears later during the power-law dominant state. The transient nature indicates that MAXI J1631-479 is most likely to be an LMXB. Therefore, the absorption feature found in MAXI J1631-479 probably arises from disk wind rather than the stellar wind from a massive companion star. It is uncommon for strong disk wind features to be detected in a low-inclination black hole binary. We note that the detection of a blueshifted ionized iron absorption feature in MAXI J1631-479 (with the disk inclination angle of $i \approx 29^{\circ}$, close to face-on) implies that the disk wind observed in MAXI J1631-479 is collimated to be nearly perpendicular to the disk or has a narrow opening angle assuming a conical geometry. The presence of disk wind supports MAXI J1631-479 accreting at a high accretion rate at the time of our NuSTAR observations. The disappearance of absorption features at the second epoch might be caused by a geometric change of the wind due to the state transition or overionization of the outflowing material due to increased hard $\mathrm{X}$-ray illumination from the central engine (e.g., Miller et al. 2006; Ueda et al. 2010; Díaz Trigo et al. 2014). It supports a change in the accretion mode between the two epochs.

In addition, we note that the disk wind velocity we find from the best-fit results is very high, $v_{\text {out }}=0.067_{-0.004}^{+0.001} c\left(20100_{-1200}^{+300}\right.$ $\mathrm{km} \mathrm{s}^{-1}$ ), significantly exceeding the typical range for disk winds detected in black hole X-ray binaries (e.g., King et al. 2013; Shidatsu et al. 2013; Díaz Trigo \& Boirin 2016). This velocity is comparable to some of the most extreme cases that have been reported in black hole X-ray binaries (Chiang et al. 2012; King et al. 2012; Xu et al. 2018b; Miller et al. 2019) and might be an analog to the ultra-fast outflows (UFOs) detected in a number of AGNs (e.g., Tombesi et al. 2010, 2013; Nardini et al. 2015).

\section{Conclusion}

In this work, we report strong and variable relativistic disk reflection features in the new black hole binary candidate MAXI J1631-479 detected by NuSTAR. Significant difference is found in the strength and the red wing of the broad iron line observed at two epochs, which caught the source in the disk dominant and the power-law dominant state. The iron line flux in the disk dominant state is unusually high when compared with the flux of the weak power-law component, and the iron line flux is not correlated with the strength of the coronal emission. These suggest that the reprocessing of high-energy disk photons plays an important role in shaping the disk reflection spectra we observe in the disk dominant state.

By fitting the NuSTAR spectra with self-consistent reflection models, the change in the line profile can be explained by an increase in the inner radius of the optically thick accretion disk: the inner edge of the accretion disk extends down to the ISCO in the disk dominant state and becomes truncated at a larger radius during the power-law dominant state. We discuss the possible physical mechanism (local disk instabilities) that could trigger disk truncation without a significant change in the luminosity/ accretion rate. In addition, the results indicate that the central object in MAXI J1631-479 is a rapidly spinning black hole with a spin parameter of $a^{*}>0.94$, and the accretion disk is viewed at a low inclination angle of $i=29^{\circ} \pm 1^{\circ}$. This is also an uncommon case where a disk wind feature is detected in a black hole X-ray binary viewed close to face-on, where the disk wind is found to be extremely fast for a black hole X-ray binary.

We thank the referee for the constructive comments that improved this paper. D.J.W. acknowledges support from an STFC Ernest Rutherford fellowship. J.A.G. acknowledges support from NASA grant NNX17AJ65G and from the Alexander von Humboldt Foundation. J.H. acknowledges support from an appointment to the NASA Postdoctoral Program at the Goddard Space Flight Center, administered by the USRA through a contract with NASA. M.L.P. is supported by the European Space Agency (ESA) Research Fellowship. This work was supported under NASA contract No. NNG08FD60C and made use of data from the NuSTAR mission, a project led by the California Institute of Technology, managed by the Jet Propulsion Laboratory and funded by the National Aeronautics and Space Administration. We thank the NuSTAR Operations, Software, and Calibration teams for support with the execution and analysis of these observations. This research has made use of the NuSTAR Data Analysis Software (NuSTARDAS), jointly developed by the ASI Science Data Center (ASDC, Italy) and the California Institute of Technology (USA). This research has also made use of MAXI data provided by RIKEN, JAXA, and the MAXI team.

\section{ORCID iDs}

Yanjun Xu (iD https://orcid.org/0000-0003-2443-3698 Fiona A. Harrison (iD https://orcid.org/0000-0003-2992-8024 John A. Tomsick (iD https://orcid.org/0000-0001-5506-9855 
Dominic J. Walton (1) https://orcid.org/0000-0001-5819-3552

Didier Barret 는 https://orcid.org/0000-0002-0393-9190

Javier A. García (i) https://orcid.org/0000-0003-3828-2448

Jeremy Hare (1) https://orcid.org/0000-0002-8548-482X

Michael L. Parker (1) https://orcid.org/0000-0002-8466-7317

\section{References}

Arnaud, K. A. 1996, in ASP Conf. Ser. 101, Astronomical Data Analysis Software and Systems V, ed. G. H. Jacoby \& J. Barnes (San Francisco, CA: ASP), 17

Bardeen, J. M., Press, W. H., \& Teukolsky, S. A. 1972, ApJ, 178, 347 Begelman, M. C., McKee, C. F., \& Shields, G. A. 1983, ApJ, 271, 70

Belloni, T., Méndez, M., King, A. R., van der Klis, M., \& van Paradijs, J. 1997, ApJL, 479, L145

Belloni, T. M., \& Motta, S. E. 2016, in Astrophysics of Black Holes: From Fundamental Aspects to Latest Developments, Astrophysics and Space Science Library, Vol. 440, ed. C. Bambi (Berlin: Springer-Verlag), 61

Brenneman, L. W., \& Reynolds, C. S. 2006, ApJ, 652, 1028

Buisson, D. J. K., Fabian, A. C., Barret, D., et al. 2019, MNRAS, 490, 1350

Cackett, E. M., Miller, J. M., Ballantyne, D. R., et al. 2010, ApJ, 720, 205

Chiang, C.-Y., Reis, R. C., Walton, D. J., \& Fabian, A. C. 2012, MNRAS, 425,2436

Corral-Santana, J. M., Casares, J., Muñoz-Darias, T., et al. 2016, A\&A, 587, A61

Dauser, T., García, J., Parker, M. L., Fabian, A. C., \& Wilms, J. 2014, MNRAS, 444, L100

Dauser, T., García, J., Walton, D. J., et al. 2016, A\&A, 590, A76

Dauser, T., Garcia, J., Wilms, J., et al. 2013, MNRAS, 430, 1694

Dauser, T., Wilms, J., Reynolds, C. S., \& Brenneman, L. W. 2010, MNRAS, 409, 1534

Davis, S. W., \& El-Abd, S. 2019, ApJ, 874, 23

Díaz Trigo, M., \& Boirin, L. 2016, AN, 337, 368

Díaz Trigo, M., Migliari, S., Miller-Jones, J. C. A., \& Guainazzi, M. 2014, A\&A, 571, A76

Done, C., \& Kubota, A. 2006, MNRAS, 371, 1216

Eijnden, J. v. d., Ludlam, R. M., Homan, J., et al. 2019, ATel, 12440, 1

Esin, A. A., McClintock, J. E., \& Narayan, R. 1997, ApJ, 489, 865

Esin, A. A., Narayan, R., Cui, W., Grove, J. E., \& Zhang, S.-N. 1998, ApJ, 505,854

Fabian, A. C., Rees, M. J., Stella, L., \& White, N. E. 1989, MNRAS, 238, 729 Fabian, A. C., \& Ross, R. R. 2010, SSRv, 157, 167

Fiocchi, M, Onori, F, Bazzano, A, et al. 2020, MNRAS, 492, 3657

Frank, J., King, A., \& Raine, D. J. 2002, Accretion Power in Astrophysics (3rd ed.; Cambridge: Cambridge Univ. Press)

Frank, J., King, A. R., \& Lasota, J. P. 1987, A\&A, 178, 137

Fürst, F., Nowak, M. A., Tomsick, J. A., et al. 2015, ApJ, 808, 122

García, J., Dauser, T., Reynolds, C. S., et al. 2013, ApJ, 768, 146

García, J. A., Dauser, T., Lohfink, A., et al. 2014, ApJ, 782, 76

García, J. A., Tomsick, J. A., Sridhar, N., et al. 2019, ApJ, 885, 48

Gierliński, M., Done, C., \& Page, K. 2008, MNRAS, 388, 753

Gu, W.-M., \& Lu, J.-F. 2000, ApJL, 540, L33

Guilbert, P. W., \& Rees, M. J. 1988, MNRAS, 233, 475

Haardt, F., \& Maraschi, L. 1991, ApJL, 380, L51

Haardt, F., \& Maraschi, L. 1993, ApJ, 413, 507

Harrison, F. A., Craig, W. W., Christensen, F. E., et al. 2013, ApJ, 770, 103

Honma, F. 1996, PASJ, 48, 77

Kallman, T., \& Bautista, M. 2001, ApJS, 133, 221

Kara, E., Steiner, J. F., Fabian, A. C., et al. 2019, Natur, 565, 198

King, A. L., Miller, J. M., Raymond, J., et al. 2012, ApJL, 746, L20

King, A. L., Miller, J. M., Raymond, J., et al. 2013, ApJ, 762, 103

Kobayashi, K., Maruyama, W., Negoro, H., et al. 2018, ATel, 12320, 1

Kong, A. K. H. 2019, ATel, 12504, 1

Krimm, H. A., Holland, S. T., Corbet, R. H. D., et al. 2013, ApJS, 209, 14

Krolik, J. H., \& Hawley, J. F. 2002, ApJ, 573, 754

Kubota, A., \& Done, C. 2004, MNRAS, 353, 980

Lightman, A. P., \& Eardley, D. M. 1974, ApJL, 187, L1

Lightman, A. P., \& White, T. R. 1988, ApJ, 335, 57

Lu, J.-F., Lin, Y.-Q., \& Gu, W.-M. 2004, ApJL, 602, L37

Ludlam, R. M., Miller, J. M., Bachetti, M., et al. 2017, ApJ, 836, 140

Ludlam, R. M., Miller, J. M., Barret, D., et al. 2019, ApJ, 873, 99

Matsuoka, M., Kawasaki, K., Ueno, S., et al. 2009, PASJ, 61, 999

McClintock, J. E., \& Remillard, R. A. 2006, in Compact Stellar X-ray Sources, Vol. 39, ed. W. Lewin \& M. van der Klis (Cambridge: Cambridge Univ. Press), 157
McClintock, J. E., Shafee, R., Narayan, R., et al. 2006, ApJ, 652, 518 Merloni, A., Fabian, A. C., \& Ross, R. R. 2000, MNRAS, 313, 193

Meyer, F., Liu, B. F., \& Meyer-Hofmeister, E. 2000, A\&A, 361, 175

Middleton, M. J., Parker, M. L., Reynolds, C. S., Fabian, A. C., \& Lohfink, A. M. 2016, MNRAS, 457, 1568

Miller, J. M. 2007, ARA\&A, 45, 441

Miller, J. M., Gendreau, K., Ludlam, R. M., et al. 2018, ApJL, 860, L28

Miller, J. M., Raymond, J., Homan, J., et al. 2006, ApJ, 646, 394

Miller, J. M., Tomsick, J. A., Bachetti, M., et al. 2015, ApJL, 799, L6

Miller, J. M., Zoghbi, A., Gandhi, P., \& Paice, J. 2019, ATel, 13012, 1

Miller, L., Turner, T. J., Reeves, J. N., et al. 2007, A\&A, 463, 131

Miniutti, G., Fabian, A. C., Goyder, R., \& Lasenby, A. N. 2003, MNRAS, 344, L22

Miniutti, G., Fabian, A. C., \& Miller, J. M. 2004, MNRAS, 351, 466

Mirabel, I. F., Dhawan, V., Chaty, S., et al. 1998, A\&A, 330, L9

Mitsuda, K., Inoue, H., Koyama, K., et al. 1984, PASJ, 36, 741

Miyasaka, H., Tomsick, J. A., Xu, Y., \& Harrison, F. A. 2018, ATel, 12340, 1

Motta, S. E., Kajava, J. J. E., Sánchez-Fernández, C., Giustini, M., \& Kuulkers, E. 2017, MNRAS, 468, 981

Nakahira, S., Shidatsu, M., Makishima, K., et al. 2018, PASJ, 70, 95

Narayan, R., \& Yi, I. 1995, ApJ, 452, 710

Nardini, E., Reeves, J. N., Gofford, J., et al. 2015, Sci, 347, 860

Park, S. Q., Miller, J. M., McClintock, J. E., et al. 2004, ApJ, 610, 378

Parker, M. L., Marinucci, A., Brenneman, L., et al. 2014, MNRAS, 437, 721

Parker, M. L., Tomsick, J. A., Kennea, J. A., et al. 2016, ApJL, 821, L6

Parker, M. L., Tomsick, J. A., Miller, J. M., et al. 2015, ApJ, 808, 9

Ponti, G., Fender, R. P., Begelman, M. C., et al. 2012, MNRAS, 422, L11

Pooley, G. G., \& Fender, R. P. 1997, MNRAS, 292, 925

Reis, R. C., Fabian, A. C., Ross, R. R., et al. 2008, MNRAS, 387, 1489

Reis, R. C., Miller, J. M., Reynolds, M. T., et al. 2013, ApJ, 763, 48

Remillard, R. A., \& McClintock, J. E. 2006, ARA\&A, 44, 49

Ross, R. R., \& Fabian, A. C. 2005, MNRAS, 358, 211

Ross, R. R., \& Fabian, A. C. 2007, MNRAS, 381, 1697

Rossi, S., Homan, J., Miller, J. M., \& Belloni, T. 2005, MNRAS, 360, 763

Russell, T. D., van den Eijnden, J., \& Degenaar, N. 2019, ATel, 12396, 1

Salvesen, G., Miller, J. M., Reis, R. C., \& Begelman, M. C. 2013, MNRAS, 431,3510

Shakura, N. I., \& Sunyaev, R. A. 1973, A\&A, 500, 33

Shidatsu, M., Nakahira, S., Murata, K. L., et al. 2019, ApJ, 874, 183

Shidatsu, M., Ueda, Y., Nakahira, S., et al. 2013, ApJ, 779, 26

Shields, G. A., McKee, C. F., Lin, D. N. C., \& Begelman, M. C. 1986, ApJ, 306, 90

Shimura, T., \& Takahara, F. 1995, ApJ, 445, 780

Steiner, J. F., McClintock, J. E., Remillard, R. A., Narayan, R., \& Gou, L. 2009a, ApJL, 701, L83

Steiner, J. F., Narayan, R., McClintock, J. E., \& Ebisawa, K. 2009b, PASP, 121,1279

Steiner, J. F., Remillard, R. A., García, J. A., \& McClintock, J. E. 2016, ApJL, 829, L22

Straub, O., Bursa, M., Sądowski, A., et al. 2011, A\&A, 533, A67

Takeuchi, M., \& Mineshige, S. 1998, ApJL, 505, L19

Tamura, M., Kubota, A., Yamada, S., et al. 2012, ApJ, 753, 65

Tetarenko, B. E., Sivakoff, G. R., Heinke, C. O., \& Gladstone, J. C. 2016, ApJS, 222, 15

Thorne, K. S. 1974, ApJ, 191, 507

Tombesi, F., Cappi, M., Reeves, J. N., et al. 2010, A\&A, 521, A57

Tombesi, F., Cappi, M., Reeves, J. N., et al. 2013, MNRAS, 430, 1102

Tomsick, J. A., Nowak, M. A., Parker, M., et al. 2014, ApJ, 780, 78

Tomsick, J. A., Parker, M. L., García, J. A., et al. 2018, ApJ, 855, 3

Tomsick, J. A., Yamaoka, K., Corbel, S., et al. 2009, ApJL, 707, L87

Ueda, Y., Honda, K., Takahashi, H., et al. 2010, ApJ, 713, 257

Verner, D. A., Ferland, G. J., Korista, K. T., \& Yakovlev, D. G. 1996, ApJ, 465,487

Walton, D. J., Mooley, K., King, A. L., et al. 2017, ApJ, 839, 110

Walton, D. J., Reis, R. C., Cackett, E. M., Fabian, A. C., \& Miller, J. M. 2012, MNRAS, 422, 2510

Walton, D. J., Tomsick, J. A., Madsen, K. K., et al. 2016, ApJ, 826, 87

Wilkins, D. R., \& Fabian, A. C. 2012, MNRAS, 424, 1284

Wilms, J., Allen, A., \& McCray, R. 2000, ApJ, 542, 914

Xu, Y., García, J. A., Fürst, F., et al. 2017, ApJ, 851, 103

Xu, Y., Harrison, F. A., García, J. A., et al. 2018a, ApJL, 852, L34

Xu, Y., Harrison, F. A., Kennea, J. A., et al. 2018b, ApJ, 865, 18

Yuan, F., \& Narayan, R. 2004, ApJ, 612, 724

Zdziarski, A. A., Johnson, W. N., \& Magdziarz, P. 1996, MNRAS, 283, 193

Życki, P. T., Done, C., \& Smith, D. A. 1999, MNRAS, 309, 561 